Revista Signos

2008, 41(68)

403-422

\title{
Teorías implícitas de los profesores acerca de la comprensión de textos
}

\author{
Margarita Makuc* \\ Universidad de Magallanes \\ Chile
}

Resumen: El objetivo de este artículo es describir los procesos de comprensión de textos a partir de las teorías implícitas de un grupo de docentes. Desde un punto de vista teórico se plantea que los sujetos elaboran teorías acerca de diversos dominios de su experiencia, las que constituirían un tipo de conocimiento implícito, que estaría en la base de las decisiones y acciones de los sujetos (Dienes \& Perner, 1999; Pozo, 2001; Rodrigo \& Correa, 2001). Para alcanzar el objetivo señalado, se utilizaron dos técnicas de recolección de datos: Focus Group y Cuestionario de Metacomprensión. El análisis de los resultados nos permitió identificar tres tipos de teorías implícitas: lineal, interactiva y transaccional. El predominio de la teoría lineal en los resultados del Focus Group y de la teoría interactiva en el Cuestionario de Metacomprensión, nos permite inferir que las teorías implícitas que orientan las acciones de los docentes varían y se adaptan a las características del contexto y a las demandas a las que se ve enfrentado, activando una teoría lineal en contextos de enseñanza, y una teoría interactiva en un contexto de lectura individual.

Palabras Clave: Teorías implícitas, comprensión textual, Focus Group, metacomprensión.

Recibido:

13-IV-2007

Aceptado:

22-VII-2008

* Becaria Proyecto MECESUP UCV/ 0004, Doctorado en Lingüística PUCV.

Correspondencia: Margarita Makuc (margarita.makuc@umag.cl) Tel.: (56-61) 207165. Fax: (56-61) 212896. Universidad de Magallanes. Avenida Bulnes 01855, Casilla 113-D, Punta Arenas, Chile. 


\section{Teachers' implicit theories of text comprehension}

This article attempts to describe text comprehension processes from teachers' implicit theories. Theoretically, it is suggested that the subjects elaborate theories of several domains of their experience. These theories constitute a sort of implicit knowledge that will be at the base of the subjects' decisions and actions (Dienes \& Perner, 1999; Pozo, 2001; Rodrigo \& Correa, 2001). To achieve this, two data gathering techniques were employed: a focus group and metacognition questionnaires. The findings help identify three implicit theories: linear, interactive, and transactional. The predominance of the linear theory in the focus group findings and of the interactive theory in those of the metacognition questionnaire help infer that implicit theories guiding teachers' actions vary and adapt to contextual characteristics and to the demands they meet, activating a linear theory in teaching contexts and an interactive theory in individual reading contexts.

Key Words: Implicit theories, textual comprehension, Focus Group, metacomprehension.

\section{INTRODUCCIÓN}

Este artículo aborda la temática de la comprensión textual a partir de la noción de teorías implícitas. Estas teorías, definidas como constructos epistémicos de representación de la realidad, se elaborarían a partir de la experiencia y reflejarían la influencia de diversos modelos culturales. Su importancia radica en que constituyen síntesis de conocimientos que los sujetos han elaborado social e individualmente respecto a un dominio específico; al respecto se plantea que estarían en la base de las decisiones y acciones de los sujetos (Dienes \& Perner, 1999; Pozo, 2001; Rodrigo \& Correa, 2001). Desde esta perspectiva, en este artículo nos interesa presentar las teorías implícitas de un grupo de docentes acerca de la comprensión y reconocer en ellas la presencia de los principales modelos teóricos que en el ámbito de la comprensión fueron identificados en la revisión bibliográfica.

Desde un punto de vista metodológico, la identificación y caracterización de las teorías implícitas que orientan las acciones de los docentes se realiza mediante dos modalidades: Focus Group y Cuestionario de Metacomprensión. La combinación de ambas estrategias, se debe a que, desde una perspectiva teórica, la diferencia entre verbalización y reconocimiento, representaría niveles de explicitación o conciencia (Karmiloff-Smith, 1994). De acuerdo a la propuesta de los elementos que constituyen las teorías implícitas, éstas se situarían al inicio del continuo representacional evolutivo desde un polo implícito a otro polo explícito. Por consiguiente, para acceder al nivel implícito, es preciso desarrollar diversas estrategias que permitan la explicitación progresiva de las teorías en cuestión. En este estudio se propone abordar el nivel E2 definido como un nivel menos explícito (o de reconocimiento) mediante el Cuestionario de Metacomprensión; y el nivel E3, esto es, de verbalización, mediante la técnica del Focus Group. 


\section{Antecedentes teóricos}

\subsection{La noción de teoría implícita como una aproximación a las teorías de los profesores sobre la comprensión de textos}

En términos generales, este constructo nos remite a un complejo conjunto de relaciones entre conceptos, usualmente con una base causal, los cuales son organizados en teorías, entendidas como entidades que forman parte de una explicación mental y no de una explicación científica. Desde esta perspectiva, los conceptos estarían insertos en teorías y modelos mentales complejos que constituirían dominios representados mentalmente de fenómenos y principios explicativos (Murphy \& Medin, 1985; Carey \& Spelke, 1993; Rodrigo, 1994a; Benlloch, 1997). Una característica central de estas teorías es que además de constituir un dispositivo epistémico de construcción de la realidad, son un tipo de entidad cognitiva, inconsciente e implícita, que ejerce una enorme influencia en la conducta y en la respuesta a determinados problemas (Groeben, 1990; Karmiloff-Smith, 1994).

Al mismo tiempo, diversas propuestas (Dienes \& Perner, 1999; Kihlstrom, 1999; Reber, Allen \& Reber, 1999; Pozo, 2001) han planteado la polaridad implícito/explícito como un continuo más que como una dicotomía. El establecimiento de distintos niveles en el continuo explícito/implícito, respondería a criterios representacionales, funcionales o conceptuales, que permitan la explicitación de los fenómenos de cognición implícita mediante procesos graduales.

La distinción entre verbalización y reconocimiento representaría niveles de explicitación que permitirían dar cuenta de nuestros conocimientos acerca de un dominio determinado. Karmiloff-Smith (1994) a través de la teoría de la redescripción representacional intenta conciliar el enfoque innatista modularista como el de enfoque piagetano acerca de la construcción generalizada del conocimiento. De esta manera, para la autora, existiría predisposición innata específica correspondiente a ciertos microdominios, que no pueden definirse como módulos encapsulados sino más bien como un "sesgo o boceto en esqueleto" (Karmiloff-Smith, 1994) que, progresivamente, debido a factores externos e internos experimentarían un proceso de modularización haciéndose menos accesibles, más automáticos e independientes de otros sistemas. La idea central de la teoría de la redescripción representacional es que junto a este proceso de encapsulamiento progresivo, se produce un proceso inverso de mayor apertura y accesibilidad. Además, gracias a procesos mediante los cuales el sujeto va cambiando el formato de representación de la información que ha almacenado, ésta pasa de niveles implícitos (conocimiento procedimental) a niveles más explícitos y más conscientes (conocimiento declarativo).

En este cambio de formato o redescripción, Karmiloff-Smith (1994) reconoce, al menos, cuatro niveles: nivel 1, al que denomina implícito y que se codificaría en forma procedimental y no estaría abierto a otros sistemas. En el dominio del lenguaje, este conocimiento se almacenaría 
como procedimientos lingüísticos que permiten (en el caso del niño) hablar y comprender el lenguaje oral pero no reflexionar sobre él. El nivel E1, se considera un nivel más abstracto que el anterior que está abierto a la formación de vínculos intra e interdominios permitiendo establecer relaciones analógicas y por tanto crear conocimientos y términos nuevos. En el dominio del lenguaje, este nivel se reflejaría en las autocorrecciones espontáneas y en la capacidad de mentir, es decir, distinguir entre palabras y las cosas del mundo extralingüístico o de comprender oraciones contrarias a los hechos. Nuevas redescripciones, en lenguajes cada vez más parecidos a las lenguas naturales llevan a los niveles E2 y E3 que permiten alcanzar un nivel consciente y verbalizable de la información, respectivamente.

Ahora bien, la búsqueda de los elementos que componen las teorías implícitas de los profesores acerca de la comprensión textual la abordaremos siguiendo la propuesta de redescripción representacional anteriormente comentada. De acuerdo a esta propuesta, los elementos que constituyen las teorías implícitas se situarían en un extremo del continuo representacional que va desde un polo implícito a otro polo explícito.

Para efectos del estudio presentado en este artículo, nos centramos en el proceso de explicitación del conocimiento (Karmiloff-Smith, 1994) llevado a cabo a partir de la realización de Focus Group y de la aplicación de un Cuestionario de Metacomprensión, como se aprecia en la siguiente figura:

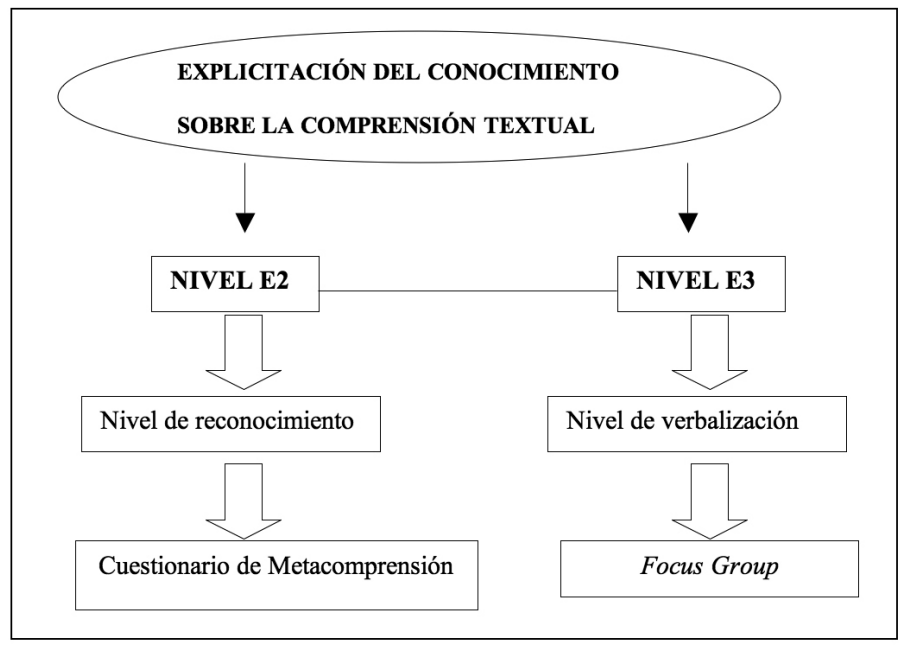

Figura 1. Esquema de la redescripción representacional (basado en Karmiloff-Smith, 1994). 


\subsection{Las teorías implícitas y el estudio del pensamiento del profesor}

El enfoque de las teorías implícitas se vincula con el paradigma definido como 'Pensamiento del Profesor' (Clark \& Peterson, 1990), pues desde este enfoque las investigaciones sobre la enseñanza se proponen analizar no sólo las acciones de los profesores, sino principalmente los procesos de pensamiento que los provocan. Esta nueva concepción de la enseñanza, y del profesor, sugiere incorporar en el análisis la perspectiva de los sujetos de la investigación, dado que concibe al profesor y al alumno como agentes activos cuyos pensamientos, planes y percepciones influyen y determinan su conducta (Marcelo, 1987). Dentro de este esquema, se plantea que el comportamiento es, en gran medida, el resultado del pensamiento del profesor, tanto de sus conocimientos como de sus estrategias para procesar la información y utilizarla en la resolución de problemas (Pérez Gómez, 1985).

Entre los aspectos que interesa abordar se ubica el estudio de las percepciones de los profesores acerca de sí mismos, su rol y la enseñanza; por ello, se delimitan tres dominios de investigación: planificación docente, toma de decisiones interactivas y teorías implícitas (Clark \& Peterson, 1990; Sandín, 2003). El dominio de investigación orientado al estudio de las teorías de los profesores, considera el conjunto de procesos básicos que ocurren en la mente del profesor cuando organiza, dirige y desarrolla su comportamiento, tanto en los momentos previos como durante la enseñanza. Este conjunto de procesos se expresaría en creencias y conocimientos sobre la enseñanza, los estudiantes y el contenido y en la conciencia de estrategias de resolución de problemas endémicos a la enseñanza.

En el contexto de este estudio, esta revisión teórica nos ha permitido desarrollar dos aspectos centrales de esta investigación: la noción de teoría implícita y los estudios acerca del pensamiento del profesor. La revisión de la noción de teoría implícita nos permitió comprobar que este constructo surgió como reacción a una concepción positivista y su sobrevaloración de lo científico como única forma de conocimiento válido; ello se expresa en el marco del paradigma del pensamiento del profesor al reconocer que los docentes construyen teorías en función de las demandas de la situación y que sus acciones didácticas están dirigidas por estas teorías aún cuando no sea de manera explícita.

Junto con la presentación de los conceptos centrales de la propuesta de teorías implícitas, en este artículo hemos incluido los aportes teóricos que han consolidado el ámbito de la comprensión textual, por cuanto esta investigación se plantea identificar los paradigmas, enfoques o modelos teóricos que pudieran estar influyendo en las concepciones de los profesores respecto de este dominio del conocimiento. Dichas teorías nos permiten reconocer los enfoques y/o modelos que han tenido mayor impacto en definir y caracterizar los procesos de comprensión textual, así como observar la confluencia de disciplinas como la lingüística, la psicología, la sociología y la filosofía. 


\subsection{Teorías acerca del dominio de la comprensión textual}

Una aproximación global a esta temática permitió identificar tres paradigmas que subyacen en el estudio de la comprensión: Conductista, Cognitivo y Socio-comunicativo (Pearson \& Stephens, 1994). Desde nuestra perspectiva de análisis, estos paradigmas se expresan, en diversos grados y formas, en cuatro teorías sobre la comprensión de textos: teoría lineal; teoría cognitiva, teoría interactiva y teoría transaccional. Estas teorías, entendidas como un conjunto de conceptos interrelacionados que describen y explican un fenómeno, se formularon a partir de los supuestos centrales en torno a la noción de comprensión, de lector y de texto.

\subsubsection{Teoría lineal de la comprensión}

Esta teoría se define como lineal, pues la lectura se tiende a concebir como un proceso perceptual directo. Los lectores se conciben como decodificadores de símbolos gráficos, los cuales deben ser traducidos a un código oral. En otras palabras, la comprensión del material escrito es, fundamentalmente, la comprensión del habla, producida en la mente del lector. En esta visión la lectura no es vista como un proceso de lenguaje, sino como un proceso perceptual, mediado por un proceso de traducción, apoyado en un código lingüístico y el cual posteriormente es tratado por el cerebro como un proceso de lenguaje (Pearson \& Stephens, 1994). Esta teoría, que predomina en una primera etapa de los estudios sobre la comprensión, según Linuesa y Domínguez (1999), habría permitido plantear la existencia de dos concepciones de la lectura: a) leer consistiría en transformar los signos gráficos en significados, enfatizando los procesos de reconocimiento de la palabra escrita y b) leer sería comprender, lo que implica que los procesos de comprensión lectora se inscriben dentro de un modelo general que serviría también para explicar el lenguaje oral. Al respecto Parodi (2003) plantea que aun cuando los primeros métodos fónicos de enseñanza no hayan estado explícitamente apoyados en una teoría, es posible considerarlos como los primeros modelos de lectura comprensiva. En esta línea se inscriben los modelos de Gough (1994) y Laberge y Samuels (1994).

\subsubsection{Teoría cognitiva de la comprensión (de orientación generativista)}

La lectura, tradicionalmente entendida como una actividad de decodificación que se enseña o se aprende, pasa a ser interpretada, desde un enfoque cognitivo, como un proceso complejo, orquestado y constructivo a través del cual los individuos construyen significados. Esta reconceptualización obedece, en gran medida, al cambio de paradigma en relación al proceso mental humano, cambio que se expresó tanto en la psicolingüística como en la psicología; la superación de la perspectiva conductista permitió abordar la discusión acerca del funcionamiento interno de la mente. De este modo, la lectura es revalorizada como una posibilidad de acceder a los procesos internos y centrar la investigación en la comprensión y la relación de este proceso con el funcionamiento cognitivo humano. 
Esta concepción cognitivista del lenguaje, plantea a la psicolingüística, la necesidad de dar respuesta a los problemas relacionados con la comprensión a partir de la aplicación de los supuestos generativos del lenguaje (Chomsky, 1974). En este esfuerzo, se visualizan dos líneas principales de investigación: una, abocada a determinar la implicancia de las teorías lingüísticas para la comprensión del lenguaje; y otra, que aborda la adquisición del lenguaje. En el ámbito de la comprensión se sugiere que el número de transformaciones necesarias para llegar de la estructura superficial de una oración hasta la estructura profunda, se relaciona con la dificultad que experimenta el usuario del lenguaje al tratar de entender la oración (Pearson \& Stephens, 1994). En este marco, la comprensión se inscribe en el contexto oracional y a partir de allí se intenta determinar la incidencia que la complejidad transformacional puede tener en el proceso de comprensión. Las propuestas de Chomsky (1974) en cuanto a su concepción del lenguaje y el modo en que se adquiere y se desarrolla producen un cuestionamiento de las tendencias dominantes en lectura y los modelos lineales deben enfrentar propuestas alternativas, de esta manera el significado desde una perspectiva generativo-transformacional no se relaciona directamente con su forma externa y requiere un conjunto de reglas de transformación que permitan acceder a las proposiciones subyacentes. Desde esta perspectiva, los supuestos de este enfoque se orientan a destacar el uso creativo que los usuarios hacen de su competencia, la visión activa y participativa del sujeto y la superación del carácter tradicionalmente pasivo asignado al lector de un texto. Sin embargo, la postura chomskiana tendría una serie de debilidades relacionadas con la fuerte influencia del innatismo, así como la consideración de que el lenguaje estaría doblemente aislado de otros procesos cognitivos (Parodi, 2003).

Es preciso distinguir entre este planteamiento cognitivo de orientación generativa y el desarrollo de propuestas interactivas de la comprensión. Estas propuestas, aún estando dentro del mismo paradigma, se distinguen de la orientación generativista, en cuanto reciben los aportes de la psicología, la inteligencia artificial y la ciencia computacional. Por otra parte, para la concepción interactiva de la comprensión es preciso superar el nivel oracional de la concepción innatista de inspiración chomskiana e integrar la preocupación por el discurso y su procesamiento cognitivo.

\subsubsection{Teoría interactiva de la comprensión}

La visión interactiva de la comprensión y de los procesos mentales ha servido de base tanto a la psicología como a la linguística para abordar los procesos del lenguaje con herramientas teóricas y metodológicas que le permiten dar respuesta a múltiples inquietudes (De Vega, 1998). Durante la década de los setenta la idea general era que los modelos interactivos caracterizaban mejor la percepción del lenguaje. Hubo numerosas demostraciones empíricas de que la estructura de "nivel superior" facilita el procesamiento de la de "nivel inferior"; es decir, en el lector se activarían procesos cognitivos generales como atención percepción y memoria; que se integra- 
rían con el nivel inferior mediante la interacción entre estructuras fonológicas, morfológicas, sintácticas y semánticas del texto. Desde esta perspectiva, la comprensión se explica a partir de la interacción entre el significado del texto y los conocimientos previos del lector. Al respecto Newmeyer (1995) señala que la comprensión es un proceso en donde los modelos interactivos parecían mejor capacitados para captar la flexibilidad característica de los sistemas perceptivos y cognitivos. En cuanto a la lectura, se plantea que los lectores realizan simultáneamente un proceso de identificación y de comprensión (Goodman \& Goodman, 1994), por lo que no existiría ninguna razón para diferenciar entre la identificación de palabras y la comprensión de frases. La lectura no podría desvincularse de la comprensión, como tradicionalmente se había considerado, pues los lectores estarían naturalmente motivados a encontrar el sentido de los textos. Otro aporte importante, dentro de esta perspectiva lo constituye la obra de Smith (1983), quien plantea que si bien la percepción es parte del proceso lector, los lectores hábiles confían mínimamente en el factor visual recurriendo principalmente al conocimiento que poseen acerca de lo que leen. En este enfoque la lectura es caracterizada como una actividad o tarea que consiste en hacer predicciones informadas; los buenos lectores se diferenciarían de los menos eficientes en su capacidad de establecer criterios razonables para hacer predicciones.

La concepción interactiva de la comprensión, sintetizando los aportes de la psicolingüística, la psicología cognitiva y la teoría de los esquemas, integra las investigaciones de Rumelhart (1997), dando inicio a un conjunto importante de estudios acerca de la comprensión. Así, se afirma que el procesamiento cognitivo durante la comprensión es de carácter descendente, es decir, el procesamiento del texto escrito se realizaría a partir de los conocimientos que el lector activa durante la lectura. Esta visión del proceso, inscrita dentro de los modelos interactivos de lectura (Colomer \& Camps, 1996) supone un lector activo que procesa la información del texto basado en sus propios esquemas o marcos conceptuales emanados de su conocimiento y experiencia del mundo.

En la actualidad, existe una serie de modelos acerca de la comprensión textual que sostienen una concepción interactiva, entre ellos se destacan las propuestas de van Dijk y Kintsch (1983), Graesser, Singer y Trabasso (1994) y Kintsch (1998). Es importante resaltar la elaboración y complejidad de estos modelos, que tanto desde un punto de vista metodológico como teórico, han abordado la comprensión textual en un nivel de análisis que permite describir gran parte de los elementos involucrados en la lectura comprensiva (Duro, 1991; Parodi, 2002, 2005). El grado de exhaustividad de estos modelos entrega una visión de la complejidad de estos procesos, así como la variedad de perspectivas que pueden identificarse en el estudio de la comprensión de textos. 


\subsubsection{Teoría transaccional de la comprensión}

El modelo transaccional de Rosenblat (1996) se presenta como una propuesta que integra perspectivas muy diversas: historia, literatura, filosofía, sociología y antropología. Los términos 'transacción' y 'transaccional' representan una concepción pragmática del lenguaje, que intenta superar el tradicional concepto de interacción, noción que se asocia al paradigma positivista y que concibe las entidades de modo independiente y separable reduciendo la realidad a dualismos irreconciliables como lo son las tradicionales dicotomías: estímulo-respuesta, sujeto-objeto, individual-social; entidades entre las cuales existiría una relación de interacción. La concepción transaccional plantea un cambio en la manera de concebir la relación con el mundo que nos rodea: "Se volvió evidente que el organismo humano es el mediador último de toda percepción del mundo o de todo sentido de la realidad" (Rosenblat, 1996: 17).

La autora adoptó este término para indicar la relación doble, recíproca, que se da entre el cognoscente y lo conocido. La relación entre lector y texto es una transacción en un circuito dinámico, fluido y variable. En este proceso se fusionan lector y texto en una síntesis única e irrepetible que constituye el significado. Para Rosenblat (1996), la lectura es un momento especial en el tiempo que reúne un lector particular con un texto particular y en circunstancias particulares que dan paso a la creación de lo que ha denominado 'poema', es decir, un nuevo texto que se distingue del texto almacenado en la memoria, que es superior al texto procesado por el lector y al propio texto.

Una concepción transaccional del lenguaje concibe todo acto de lectura como un acontecimiento o una transacción que implica a un lector y un patrón de signos en particular. Desde esta perspectiva, lector y texto son aspectos de una situación dinámica total, en la cual el significado no existe de antemano en el texto o en el lector, sino que se despierta o adquiere entidad durante la transacción entre lector y texto. El texto tiene la potencialidad de evocar significados, pero no tiene el significado en sí mismo; pues éste es representado por un escritor en un texto y construido desde un texto por un lector (Goodman, 1994).

A modo de síntesis, podemos señalar que, aun cuando la concepción transaccional e interactiva comparte supuestos constructivistas respecto de la comprensión, ambos enfoques representan posturas teóricas claramente diferenciables. Por su parte, la concepción transaccional integra áreas provenientes de la historia, la literatura, la filosofía, la sociología y la antropología; mientras que el enfoque interactivo sintetiza los aportes de la psicolingüística, la psicología y la lingüística (Parodi, 2005). Por otra parte, el enfoque transaccional destaca los factores asociados al contexto durante los procesos de comprensión, señalando que el significado del texto dependerá de los contextos específicos en que se produzcan las transacciones entre lector y texto. De este modo, el enfoque transaccional enfatiza las determinantes contextuales tanto históricas, como 
socio-culturales que intervienen en la construcción de significados de los sujetos que forman parte de una comunidad cultural y social.

Como se ha señalado anteriormente, y a diferencia del enfoque transaccional, la concepción interactiva considera la comprensión de textos escritos como un proceso mental, constructivo e intencionado en que el lector elabora una interpretación de los significados del texto basándose en la información del texto escrito y sus conocimientos previos (Parodi, 2003, 2005). El enfoque interactivo, por tanto, destaca el rol activo y participativo del lector, aun cuando la lectura se produzca en un contexto determinado y obedezca a demandas del entorno.

\section{Metodología}

\subsection{Instrumentos}

Dado que esta investigación es de carácter cualitativo descriptivo, se utilizan dos instrumentos para abordar las teorías de los profesores sobre la comprensión de textos: Focus Group y Cuestionario de Metacomprensión. En términos cualitativos (Taylor \& Bogdan, 1996; Rodríguez \& Flores, 1996), se aplicó la técnica del Focus Group (Krueger, 1991; Mella, 2000) entre 18 profesores divididos en tres grupos con la finalidad de obtener información acerca de las nociones de comprensión, lector y texto. Cabe señalar, que este tipo de "conocimiento verbalizable", es definido por Karmiloff-Smith (1994) como nivel E3 e implica en el continuo de lo implícito a lo explícito un grado superior de conciencia, por cuanto permite al sujeto explicitar sus propias nociones acerca de un dominio determinado, en nuestro caso, el dominio de la comprensión textual.

Además, se aplicó a los profesores un cuestionario de metacomprensión (Peronard, Velásquez, Crespo \& Viramonte, 2002), que nos permitió acceder a una dimensión del conocimiento más cercano al nivel implícito y que ha sido definido como nivel E2 (Karmiloff-Smith, 1994). Este nivel corresponde a procesos de reconocimiento mediante los cuales los profesores deben identificar situaciones relacionadas con la comprensión de un texto, sin requerir un nivel de explicitación como el conocimiento obtenido durante los Focus Group.

Una vez realizado el análisis del Focus Group y del cuestionario en términos de las teorías predominantes, se identifican relaciones significativas entre las teorías de la comprensión que los profesores activan y explicitan tanto en el nivel declarativo durante el Focus Group, como en el nivel de reconocimiento al responder el Cuestionario de Metacomprensión.

Como ya hemos señalado, la revisión bibliográfica nos permitió proponer cuatro teorías sobre la comprensión: teoría lineal, teoría cognitiva, teoría interactiva y teoría transaccional. Estas teorías constituyen un marco de referencia y una pauta de análisis elaborada con la finalidad de interpretar y categorizar las teorías implícitas de los docentes de acuerdo a tres sub-dominios 
fundamentales en el ámbito de la comprensión: noción de comprensión, noción de lector y noción de texto.

\subsection{Resultados obtenidos en el Focus Group}

A modo de síntesis, podemos señalar que el análisis de las proposiciones extraídas de las conversaciones con los profesores en relación a las nociones de comprensión, lector y texto ha permitido identificar la influencia de tres teorías científicas sobre la comprensión de textos: teoría lineal, teoría interactiva y teoría transaccional.

Basándonos en la teoría lineal resumida previamente, hemos considerado que los docentes adhieren a ella, en tanto describen la comprensión como una actividad que consiste, principalmente, en localizar información del texto y retenerla en la memoria con la finalidad de transmitirla con fidelidad. Para los profesores que sostienen una teoría lineal sobre la comprensión, el léxico es un elemento clave en la comprensión del texto. En consecuencia, la evaluación se orienta a detectar en el alumno la información retenida del texto y, en este contexto, se concibe a un lector competente a aquel sujeto capaz de transmitir por escrito o expresar en forma oral los contenidos e ideas del texto. En esta línea se inscriben, el recurrir constantemente a evaluaciones que implican síntesis de lecturas, identificación de ideas principales, el relato y descripción de hechos. Con respecto al texto, desde una perspectiva lineal los docentes consideran que éste posee toda la información necesaria para la comprensión. Desde esta perspectiva el significado está en el texto y la función del lector es descubrirlo, podemos observar una sobrevaloración de lo textual y lo literal para el logro de una comprensión plena. Como se puede observar en (1), algunas de las afirmaciones de los docentes plantean:

(1) "comprender es interpretar 100\% lo que el autor dice"; "Yo les doy siempre algunas instrucciones, por ejemplo les digo: "en la medida que van apareciendo los personajes vayan anotándolos y van anotando qué le pasa a esa persona... pero les digo que lo hagan en una hoja anexa y vayan poniendo esos datos, entonces así en el momento que yo les pregunte qué pasaba con esto, ellos lo van a tener clarito, porque ellos ya fueron haciendo como su propio mapa de qué es lo que pasaba".

Por su parte, los docentes con una concepción transaccional se muestran proclives a promover la construcción de significados en contextos de intercambio social real, las estrategias predominantes son aquellas que enfatizan el componente comunicativo en sus diferentes modalidades: diálogos, representaciones, debates (entre otros aspectos). En esta teoría, lector competente es aquel capaz de intercambiar significados con otros sujetos en situaciones comunicativas que así los permitan, tales como: diálogo, conversaciones y discusiones grupales. Igualmente, los procedimientos de evaluación privilegian la socialización de los contenidos, los alumnos son eva- 
luados en función de su capacidad para compartir y comunicar a sus compañeros y a su profesor las ideas del texto, por lo tanto se promueve la evaluación oral. También se realizan actividades en donde el alumno transforme la modalidad del texto, ya sea con dramatizaciones, debates o diálogos, como se lee en (2):

(2) "Que en una conversación con el profesor compartan su interpretación y además que eso que leyó en algún momento de conversación, él lo saque, ¿no es cierto?, o lo converse, lo discuta, lo analice. Fíjate que es como reproducir algo con otra manera de expresión lingüística, digámoslo así, a lo mejor ponte tú una dramatización obviamente rescatando lo medular y ahí me doy cuenta si realmente han comprendido o si seleccionaron bien lo más importante, puede ser una representación o un diálogo, es decir, que ellos trasladen o transformen un poco la modalidad del texto a otra, estoy pensando justamente en lo que pudiese ser más entretenido ¿ya?”

Respecto de la teoría interactiva solo hemos considerado que un profesor adhiere claramente a sus supuestos pues resalta el papel activo y constructivo del lector y destaca la dimensión creativa de la comprensión textual, en el cual el conocimiento previo y las experiencias de vida de los alumnos son considerados aspectos centrales en la comprensión. Desde esta perspectiva, la principal estrategia consiste en promover la lectura de diferentes tipos de textos, frente a los cuales el alumno debe auto-interrogarse permanentemente acerca de cómo lo está comprendiendo. Esta teoría constituiría una clara superación del análisis literal, involucrando al mismo tiempo procesos de metacomprensión que presuponen un lector estratégico, que vaya más allá del rol asignado por una concepción lineal de la comprensión, donde la rigurosidad y la fidelidad con el texto es lo predominante. Estas estrategias promueven la integración por parte del lector de la información contenida en el texto con sus conocimientos y experiencias previas. En este enfoque se destacan aspectos no considerados en una teoría lineal ni transaccional, como son la coherencia y la cohesión, al igual que las inferencias que puedan realizar los alumnos acerca de los temas que se plantean a través de las diferentes lecturas. En definitiva, en este enfoque el texto se concibe como un medio que le permite al profesor alcanzar objetivos distintos a la reproducción del contenido o a la socialización de ellos. De este modo, como vemos en (3), se plantea:

(3) "Comprenden en la medida en que tengan conocimientos previos, ideas previas que puedan confrontar con lo que leen... el conocimiento previo y la experiencia del joven es muy importante"; "Que el alumno relacione la realidad del texto con su realidad, que genere ideas propias y que éstas sean coherentes y cohesionadas, eso es lo que me importa que haya coherencia y cohesión en lo que yo expreso... no, no espero reproducción de lo que dice tal o cual, no, aparte de que hay que respetar también lo que genera el texto en cada persona, eso es teórico pero también es una realidad, es decir, hay tantas posibles lecturas como lectores, ahora dónde está el gran desafío nuestro, en descubrir la coherencia que están manifestando los alumnos". 
En la Tabla 1, a continuación, se presenta una caracterización sintetizada de las afirmaciones de los docentes respecto a cada una de las categorías del estudio, noción de comprensión, de lector y de texto. Estos conceptos y sus respectivas descripciones los hemos organizado en términos de las teorías implícitas identificadas en este estudio.

Tabla 1. Síntesis de las teorías implícitas de los profesores según la noción de comprensión, lector y texto.

\begin{tabular}{|l|l|l|l|}
\hline & \multicolumn{1}{|c|}{ Teoría lineal } & \multicolumn{1}{c|}{ Teoría Transaccional } & \multicolumn{1}{c|}{ Teoría Interactiva } \\
\hline $\begin{array}{l}\text { Noción de } \\
\text { comprensión }\end{array}$ & $\begin{array}{l}\text { Comprender es } \\
\text { reproducir el signifi- } \\
\text { cado del texto con la } \\
\text { mayor fidelidad. }\end{array}$ & $\begin{array}{l}\text { Comprender es ser capaz de } \\
\text { compartir el significado del } \\
\text { texto a través de la comuni- } \\
\text { cación con otros. }\end{array}$ & $\begin{array}{l}\text { Comprender es interactuar con el } \\
\text { texto, construir el significado del } \\
\text { texto a partir de los conocimientos } \\
\text { previos y las experiencias. }\end{array}$ \\
\hline $\begin{array}{l}\text { Noción de } \\
\text { lector }\end{array}$ & $\begin{array}{l}\text { El lector debe } \\
\text { extraer el significado } \\
\text { del texto. }\end{array}$ & $\begin{array}{l}\text { El lector en la interacción } \\
\text { con los otros construye el } \\
\text { significado del texto. }\end{array}$ & $\begin{array}{l}\text { El lector es activo, a través de la } \\
\text { lectura integra los significados del } \\
\text { texto con } \\
\text { su experiencia y conocimientos. }\end{array}$ \\
\hline $\begin{array}{l}\text { Noción de } \\
\text { texto }\end{array}$ & $\begin{array}{l}\text { El texto entrega } \\
\text { toda la información } \\
\text { necesaria para la } \\
\text { comprensión. El } \\
\text { vocabulario del texto } \\
\text { es fundamental para } \\
\text { su comprensión. }\end{array}$ & $\begin{array}{l}\text { El texto debe ser reestruc- } \\
\text { turado, transformado y } \\
\text { comunicado en otras formas } \\
\text { orales o escritas. }\end{array}$ & $\begin{array}{l}\text { El texto es una parte importante, } \\
\text { pero su significado se completa } \\
\text { con el que el lector es capaz de } \\
\text { asignarle. El vocabulario no es } \\
\text { tan relevante. Aspectos como la } \\
\text { coherencia y la cohesión facilitan } \\
\text { la comprensión. }\end{array}$ \\
\hline
\end{tabular}

En la Tabla 1, podemos ver que la teoría cognitiva (de orientación generativista) no se manifiesta en ninguna de las proposiciones analizadas. En cambio sí ha surgido una teoría que no fue considerada previamente y que hemos definido como teoría "emergente". Esta ha sido definida como teoría literaria, ya que tanto en la noción de comprensión como en la caracterización del lector y del texto predominan criterios relacionados con una lectura literaria, es decir, con un tipo de comprensión orientada a textos literarios, ya sea textos líricos o en prosa, clásicos o contemporáneos con la finalidad explícita de estimular la apreciación estética de dichas obras. Consecuentemente, en esta teoría de la comprensión textual se destaca la dimensión lúdica y de entretención que implica la posibilidad de disfrutar con la lectura, resaltando aspectos relacionados con lo afectivo-actitudinal. En este enfoque, un buen lector es definido como aquel que es capaz de analizar los textos de acuerdo a parámetros estético-literario convencionales, y a su vez, incorporar en ese análisis su propia valoración estética. En el marco de esta teoría, los profesores tienden a percibir que un 'vocabulario restringido' del lector obstaculiza este proceso de apreciación y en consecuencia no le permitirían 'sintonizar con el cuento'. Uno de los principales desafíos de los profesores que sustentan una 'teoría implícita literaria' está rela- 
cionado con la selección de textos atractivos para el estudiante y que estén conectados con sus vivencias, tal como puede verse en el planteamiento del Ejemplo (4):

(4) "No hay entretención, no hay goce en la lectura porque no está la comprensión, al no comprender no puedes tú tampoco ponerte en el lugar de otro, ¿no es cierto? No hay esa empatía ponte tú con el personaje, qué sé yo porqué no hay comprensión", "que ellas se den cuenta que los temas con los clásicos todavía se pueden aplicar a lo que ellas viven en su diario vivir y de esa forma se crea un aprendizaje más significativo y a ellos no se les hace tan difícil para su comprensión".

En la Tabla 2, la 'teoría implícita literaria' se ha sintetizado en torno a los tres conceptos centrales que la constituyen:

Tabla 2. 'Teoría implícita literaria'. Teoría emergente según la noción de comprensión, lector y texto.

\begin{tabular}{|l|l|}
\hline $\begin{array}{l}\text { Noción de com- } \\
\text { prensión }\end{array}$ & $\begin{array}{l}\text { Comprender es imaginar, disfrutar, valorar estéticamente un texto. En esta teoría, } \\
\text { comprender implica comparar lecturas, personajes, identificar características } \\
\text { asociadas a esos personajes. }\end{array}$ \\
\hline Noción de lector & El lector disfruta con la lectura, se compromete, es un lector empático. \\
\hline Noción de texto & $\begin{array}{l}\text { El texto debe ser capaz de emocionar, debe tratar temas de interés del lector, debe } \\
\text { motivar y provocar cambios en el lector, debe provocar placer y entusiasmo en el } \\
\text { lector, debe invitar a la lectura. }\end{array}$ \\
\hline
\end{tabular}

\subsection{Presentación de resultados obtenidos en el cuestionario de metacomprensión}

Los resultados obtenidos en la aplicación del cuestionario permitieron identificar la totalidad de las teorías de base propuestas para clasificar los datos. Sin embargo, solo tres teorías reciben una manifiesta adhesión en las respuestas de los profesores: la teoría interactiva, la teoría del traspaso del contenido al lector y la teoría de índole afectiva y actitudinal.

El análisis de los resultados nos muestra que un $34,48 \%$ de las respuestas se agrupa en el puntaje 30, que a su vez representa a una teoría interactiva-comunicativa; igualmente la teoría del 'traspaso del contenido al lector' concentra un $29,21 \%$ de las respuestas, las cuales estaban asignadas con el puntaje 20. En tercer lugar, se ubican las respuestas con puntaje 12, que corresponde a una teoría de índole afectivo-actitudinal: esta teoría obtiene un 18,93\% de respuestas. Finalmente, y con una diferencia significativa en relación a los anteriores resultados, solo el 6,44\% de las respuestas se distribuyen en una teoría basada en lo externo (puntaje 9), igualmente el 5,93\% de las respuestas se ubicó en una teoría superficial e imprecisa. Finalmente, un mínimo porcentaje de respuestas (solo el 3,83\%) seleccionó una alternativa asignada con el puntaje 1 que indica ausencia de teoría. 
En el Gráfico 1 presentamos la distribución de las respuestas obtenidas por los profesores, de acuerdo a cada uno de los puntajes asignados a las respuestas del cuestionario.

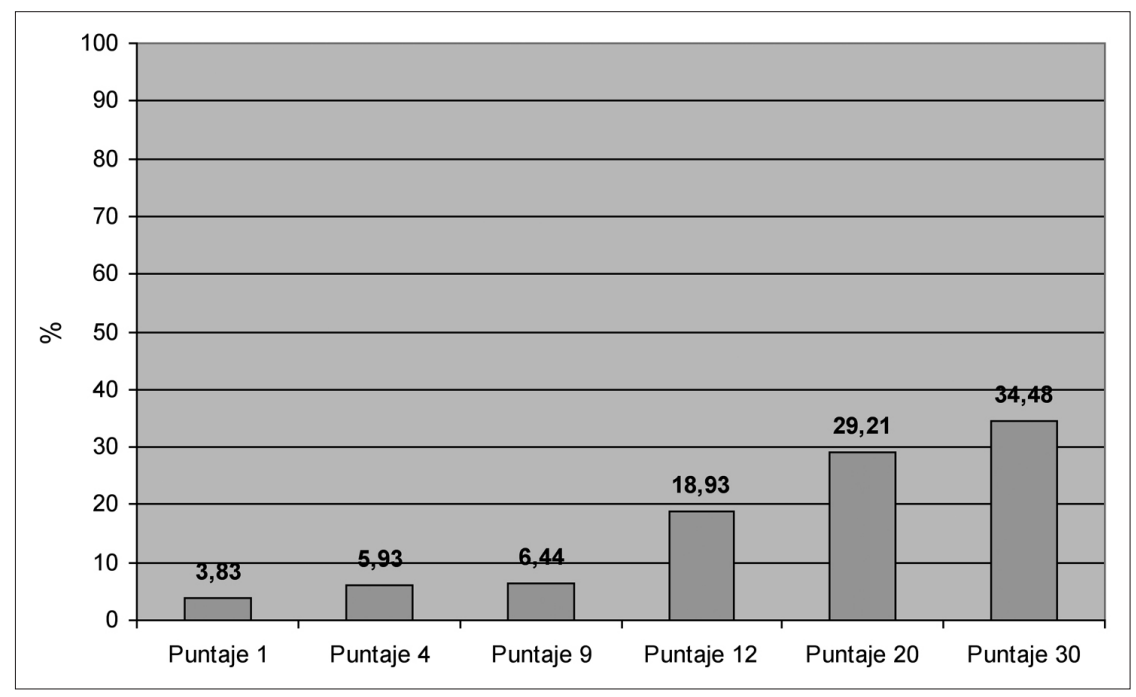

Gráfico 1. Porcentaje de respuestas del Cuestionario de Metacomprensión.

El análisis de los resultados nos permitió identificar los componentes del cuestionario que obtienen los puntajes más altos. Con respecto al primer componente (conocimiento de la tarea) encontramos que un 76\% de respuestas se ubica entre los puntajes 30/20. En segundo lugar, se ubican los puntajes obtenidos en los temas relacionados con el lector, ya que un $66 \%$ de las respuestas se ubica en estos niveles. Sin embargo, esta tendencia disminuye notoriamente en la teoría del texto, ya que las respuestas que obtienen entre 20 y 30 puntos abarcan un $48 \%$ del total de las preguntas. El análisis de cada componente nos muestra que en el conocimiento de la tarea predomina una teoría interactiva; en la teoría del lector, predomina una teoría del traspaso, y finalmente en el conocimiento del texto predomina la teoría afectiva actitudinal. Lo anterior muestra que la teoría interactiva se manifiesta claramente sólo en el ámbito de la lectura comprensiva, reflejando con ello un conocimiento estratégico de la lectura y altos índices de experticia.

En la Tabla 3 se presentan los resultados obtenidos por los docentes en los tres componentes del cuestionario, en que los puntajes obtenidos en cada componente se relacionan con las teorías correspondientes: 
Tabla 3. Resultados según componentes del Cuestionario de Metacomprensión.

\begin{tabular}{|l|c|c|c|c|c|c|}
\hline $\begin{array}{l}\text { Teorías de la } \\
\text { comprensión }\end{array}$ & $\begin{array}{c}\text { Teoría inte- } \\
\text { ractiva (30) }\end{array}$ & $\begin{array}{c}\text { Teoría del } \\
\text { traspaso } \\
(20)\end{array}$ & $\begin{array}{c}\text { Teoría afec- } \\
\text { tiva } \\
(12)\end{array}$ & $\begin{array}{c}\text { Teoría } \\
\text { basada en lo } \\
\text { externo } \\
(9)\end{array}$ & $\begin{array}{c}\text { Teoría rudi- } \\
\text { mentaria } \\
(4)\end{array}$ & $\begin{array}{c}\text { Ausencia de } \\
\text { teoría } \\
(1)\end{array}$ \\
\hline $\begin{array}{l}\text { Conocimiento } \\
\text { de la tarea }\end{array}$ & $52,08 \%$ & $24,30 \%$ & $7,63 \%$ & $5,55 \%$ & $6,94 \%$ & $1,38 \%$ \\
\hline $\begin{array}{l}\text { Teoría del } \\
\text { lector }\end{array}$ & $30,55 \%$ & $35,56 \%$ & $18,62 \%$ & $5,45 \%$ & $6,71 \%$ & $2,5 \%$ \\
\hline $\begin{array}{l}\text { Conocimiento } \\
\text { del texto }\end{array}$ & $20,83 \%$ & $27,77 \%$ & $30,55 \%$ & $8,33 \%$ & $4,16 \%$ & $7,63 \%$ \\
\hline Total & $34,48 \%$ & $29,21 \%$ & $18,93 \%$ & $6,44 \%$ & $5,93 \%$ & $3,83 \%$ \\
\hline
\end{tabular}

En la Tabla 3 observamos que los resultados difieren dependiendo del componente de cada teoría; por ejemplo, en el ámbito del conocimiento de la tarea predomina la teoría interactiva, pues un 52,08\% de las respuestas se ubica en el puntaje 30; en cuanto al lector, predomina la teoría del traspaso con un $35,56 \%$ de respuestas con puntaje 20 ; mientras que en el conocimiento del texto el $30,55 \%$ de las respuestas se ubica en el puntaje 12 que corresponde a la teoría afectiva. No obstante estas diferencias observadas en los tres dominios, podemos destacar que la teoría interactiva es la teoría que obtiene un porcentaje mayor de respuestas del cuestionario $(34,48 \%)$, en segundo lugar se ubica la teoría del traspaso con $29,21 \%$ de respuestas y en tercer lugar la teoría afectiva con $18,93 \%$ de respuestas.

De acuerdo a los descriptores del cuestionario, en las respuestas de los profesores se observa el predominio de una teoría de la lectura desde una visión compleja, flexible y ajustada a los objetivos perseguidos. Igualmente, este conocimiento metalingüístico se expresa en una teoría del lector que caracteriza a los profesores como lectores expertos que mayoritariamente seleccionan las estrategias adecuadas a la situación de lectura.

\section{Comentarios de cierre}

Como hemos señalado anteriormente, nuestra pauta de análisis de los Focus Group consideraba cuatro teorías sobre la comprensión: teoría lineal, teoría cognitiva, teoría interactiva y teoría transaccional. Estas teorías, propuestas a partir de la revisión bibliográfica, nos permitieron determinar que los docentes manifiestan concepciones relacionadas con solo tres teorías: teoría lineal, teoría interactiva y teoría transaccional. Observamos que la teoría lineal ejerce una influencia importante en sus planteamientos, en tanto se reconoce como central en la comprensión, la identificación y memorización de información extraída del texto. Un hecho interesante 
de destacar es que los principios de la teoría cognitiva no se explicitaron entre los planteamientos de los docentes, y sin embargo, surgió entre sus propuestas una teoría que hemos considerado como 'emergente' y que hemos definido como 'teoría implícita literaria'. En este ámbito, se pueden identificar resultados coincidentes con diversos estudios en el ámbito de las concepciones de docentes respecto de la comprensión de textos (Rubio, 1998; Crespo, 2001).

El análisis de los resultados en términos comparativos nos permite señalar el predominio de teorías diferentes según el contexto en que éstas se activen; una teoría lineal, surgida a partir de contextos de enseñanza y una teoría interactiva comunicativa, en el contexto del profesor como lector. Lo anterior constituye un aspecto interesante, que surge de los diversos niveles abordados en este estudio (nivel declarativo de verbalización explícita y un nivel de reconocimiento cercano al nivel implícito). Los datos del Focus Group nos indican que el profesor, en su rol de maestro, describe la comprensión de textos como un proceso lineal; mientras que los resultados del Cuestionario de Metacomprensión nos señalan que el docente en su rol de lector, representa la comprensión como un proceso interactivo comunicativo.

Por otra parte, el hecho de que estas teorías surjan desde ámbitos diferenciados; un ámbito social, que estaría definido por su rol profesional; y un ámbito individual, definido por su rol como lector, nos remite a un rasgo que caracteriza las teorías implícitas (Rodrigo; 1994b, 1997, 2001), y es que estas teorías constituirían representaciones situacionales y dinámicas (JohnsonLaird, 1987, 1999) que se activan en situaciones específicas frente a demandas concretas de una situación; de este modo el profesor activa una teoría lineal en la situación de la sala de clases y una teoría interactiva en una situación de lectura individual.

Este estudio nos muestra que el profesor en el contexto de la enseñanza ajusta o modifica su teoría de la comprensión a las características del contexto y a las demandas a las que se ve enfrentado. La necesidad de interactuar satisfactoriamente con las exigencias de su entorno (disciplina, tiempo, motivación, recursos, etc.), favorecería el desarrollo de una secuencia de rutinas propias de una teoría lineal. El desarrollo de estas rutinas en las decisiones interactivas es un rasgo destacado por diversos estudios acerca del pensamiento del profesor (Shavelson \& Stern, 1985; Marcelo, 1987, 1992; Calderhead, 1988).

Por último, creemos que esta investigación puede, además, proyectarse en términos metodológicos orientados a la aplicación adaptada del Cuestionario de Metacomprensión de Peronard et al. (2002), a futuras situaciones referidas específicamente al conocimiento metacomprensivo del profesor en contextos de enseñanza. Ello permitiría, por una parte, aumentar la información acerca de las teorías implícitas que orientan las acciones de los profesores y, por otra, ampliar y especificar el alcance de los resultados de esta investigación. 


\section{REFERENCIAS BIBLIOGRÁFICAS}

Benlloch, M. (1997). Desarrollo cognitivo y teorías implícitas en el aprendizaje de las ciencias. Madrid: Visor.

Calderhead, J. (1988). Conceptualización e investigación del conocimiento profesional de los profesores. En L. Villar \& R. Venegas (Eds.), Conocimiento, creencias y teorías de los profesores (pp. 21-39). España: Marfil.

Carey, S. \& Spelke, E. (1993). Domain specific knowledge and conceptual change. En S. Goldman \& L. Hirschfeld (Eds.), Cultural knowledge and domain specificity. New York: Cambridge University Press.

Clark, C. \& Peterson, P. (1990). Los procesos de pensamiento de los docentes. En M.C. Wittrock (Ed.), Investigación de la enseñanza III. Profesores y alumnos (pp. 443-539). Madrid: Paidós.

Colomer, T. \& Camps, A. (1996). Enseñar a leer, enseñar a comprender. Madrid: Celeste Ediciones.

Crespo, N. (2001). La construcción del concepto de lectura en el interior del aula de lengua. Onmazein, 6, 223-238.

Chomsky, N. (1974). Estructuras sintácticas. México D.F.: Siglo XXI.

De Vega, M. (1998). La psicología cognitiva: Ensayo sobre un paradigma en transformación. Anuario de Psicología, 29(1), 20-44.

Dienes, Z. \& Perner, J. (1999). A theory of implicit and explicit knowledge. Behavioral and Brain Sciences, 22, 735-808.

Duro, A. (1991). La coherencia textual en los modelos para la comprensión de textos: Kintsch et al. Vs. Sanford y Garrod. Cognitiva, 4 (2), 226-242.

Goodman, Y. \& Goodman, K. (1994). To err is human: Learning about language processes by analyzing miscues. En H. Singer \& B. Rudell (Eds.), Theoretical models and processes of reading (pp. 104-123). Newark: IRA \& Erlbaum.

Goodman, K. (1994). Reading, writing, and written texts: A transactional sociophycholinguistic view. En H. Singer \& B. Rudell (Eds.), Theoretical models and processes of reading, (pp. 1093-1130). Newark: IRA \& Erlbaum.

Gough, P. (1994). One second of reading. En H. Singer \& B. Rudell (Eds.) Theoretical models and processes of reading, (pp. 661-686). Newark, Delaware: IRA \& Erlbaum.

Graesser, A., Singer, M. \& Trabasso, T. (1994). Constructing inferences during narrative text comprehension. Pyschological review, 101(3), 371-395.

Groeben, N. (1990). Subjective theories and the explanation of human action. En G.R. Semin \& K. Gergen (Eds.), Every day understanding. Social and scientific implications (pp. 19-44). London: Sage.

Johnson-Laird, P. (1987). Modelos mentales en la ciencia cognitiva. En D. Norman (Ed.) Perspectivas de la ciencia cognitiva (pp.179-231). Barcelona: Paidós. 
Johnson-Laird, P. (1999). Formal rules versus mental models in reasoning. En R. Sternberg (Ed.), The nature of cognition (pp. 587-624). Cambridge: MIT Press.

Karmiloff-Smith, A. (1994). Más allá de la modularidad. Madrid: Alianza.

Kihlstrom, J. (1999). Conscious versus unconscious cognition. En R. Sternberg (Ed.), The Nature of Cognition (pp. 173-241). Cambridge: MIT.

Kintsch, W. (1994). The role of knowledge in discourse comprehension: A construction-integration model. En H. Singer y B. Rudell (Eds.), Theoretical Models and Processes of Readin, (pp. 951995). Newark, Delaware: IRA \& Erlbaum.

Krueger, R. (1991). El grupo de discusión. Guía práctica para la investigación aplicada. Madrid: Pirámide.

Laberge, D. \& Samuels, S. J. (1994). Toward a theory of automatic information processing of reading. En H. Singer \& B. Rudell (Eds.), Theoretical models and processes of reading (pp. 689-718). Newark: IRA y Erlbaum.

Linuesa, M. \& Domínguez, A. (1999). La enseñanza de la lectura. Enfoque psicolingüístico y sociocultural. Madrid: Pirámide.

Marcelo, C. (1987). El pensamiento del profesor. Barcelona: CEAC.

Marcelo, C. (1992). La investigación sobre la formación del profesorado. Métodos de investigación y análisis de datos. Argentina: CINCEL.

Mella, O. (2000). Grupos focales. Focus Group. Técnica de investigación cualitativa. Santiago: CIDE.

Murphy, G. \& Medin, D. (1985). The role of theories in conceptual coherence. Psychological Review, 97(3), 289-316.

Newmeyer, F. (1995). Panorama de la lingüística moderna de la Universidad de Cambridge. III El lenguaje. Aspectos Psicológicos y Biológicos. Madrid: Visor.

Parodi, G. (2002). Comprensión lingüística: ¿Hacia dónde vamos desde dónde estamos? En G. Parodi (Ed.), Lingüística e interdisciplinariedad: Desafíos del nuevo milenio. Ensayos en honor a Marianne Peronard (pp. 67-93). Valparaíso: Ediciones Universitarias de Valparaíso.

Parodi, G. (2003). Relaciones entre lectura y escritura: Una perspectiva cognitiva discursiva. Valparaíso: Ediciones Universitarias de Valparaíso.

Parodi, G. (2005). Comprensión de textos escritos. Buenos Aires: EUDEBA.

Pearson, D. \& Stephens, D. (1994). Learning about literacy: A 30-year journey. En H. Singer \& B. Rudell (Eds.), Theoretical models and processes of reading (pp. 22-103). Newark: IRA \& Erlbaum.

Pérez Gómez, A. (1985). Paradigmas contemporáneos de investigación didáctica. En J. Gimeno Sacristán \& A. Pérez Gómez (Eds.), La enseñanza: Su teoría y su práctica (pp. 95-139). Madrid: Akal.

Peronard, M., Velázquez, M., Crespo, N. \& Viramonte, M. (2002) Conocimiento metacognitivo del 
lenguaje escrito: Instrumento de medida y fundamentación teórica. Infancia y aprendizaje, 25(2), 131-145.

Pozo, J. (2001). Humana mente: El mundo, la conciencia y la carne. Madrid: Morata.

Reber, A., Allen, R. \& Reber, P. (1999). Implicit versus explicit learning. En R. Sternberg (Ed.) The nature of cognition (pp. 475-505). Cambridge: MIT.

Rodrigo, M. J. (1994a). Etapas, contextos, dominios y teorías implícitas en el conocimiento social. En M. J. Rodrigo (Ed.), Contexto y desarrollo social (pp. 26-46). Madrid: Síntesis.

Rodrigo, M. J. (1994b). El hombre de la calle, el científico y el alumno: ¿un solo constructivismo o tres? Investigación en la escuela, 23 (pp. 7-16).

Rodrigo, M. J. (1997). Del escenario sociocultural al constructivismo episódico: Un viaje al conocimiento escolar de la mano de las teorías implícitas. En M.J. Rodrigo \& J. Arnay (Comps.), La construcción del conocimiento escolar (pp. 177-191). Barcelona: Paidós.

Rodrigo, M. J. \& Correa, N. (2001). Representación y procesos cognitivos: Esquemas y modelos mentales. En C. Coll, J. Palacios \& A. Marchesi (Comps.), Desarrollo psicológico y educación, 2. Psicología de la educación escolar (pp. 117-135). Madrid: Alianza.

Rodríguez, G. \& Flores, L. (1996). Metodología de la investigación cualitativa. Madrid: Ediciones Aljibe.

Rosenblatt, L. (1996). La teoría transaccional de la lectura y la escritura. En Textos en contexto. Los procesos de lectura y escritura (pp. 13-70). Buenos Aires: Proyecto Editorial Lectura y Vida.

Rubio, E. (1998). Lengua y lectura: una reflexión desde la práctica. Madrid: Editorial Escuela Española.

Rumelhart, D. (1997). Hacia una comprensión de la comprensión. En E. Rodríguez \& E. Laguer (Comp.), La lectura (pp. 25-51). Santiago de Cali: Editorial Universidad del Valle.

Sandín, M. (2003). Investigación cualitativa en educación. Madrid: McGraw-Hill.

Shavelson, R. \& Stern, P. (1985). La investigación sobre el pensamiento pedagógico del profesor, sus juicios, decisiones y conducta. En J. Gimeno Sacristán \& A. Pérez Gómez (Eds.), La enseñanza: Su teoría y su práctica (pp. 372-419). Madrid: Akal.

Smith, F. (1983). Comprensión de la lectura. Análisis psicolingüístico de la lectura y su aprendizaje. México: Trillas.

Taylor, S. \& Bogdan, R. (1996). Introducción a los métodos cualitativos de investigación. Buenos Aires: Paidós.

Van Dijk, T. \& Kintsch, W. (1983). Strategic of discourse comprehension. New York: Academic Press. 